\title{
Editorial
}

\section{Optional quality - a terminal condition!}

Ensuring the quality of medical practice involves setting standards for care against which current practice can be compared and implies that where discrepancies are found practice will be changed to meet the standards. These activities are known collectively as medical audit. Medical audit came to prominence in Britain in 1989 when the government made clear that doctors were expected to take part in the 'systemic critical analysis of the quality of medical care'.

Despite the high profile given to audit and the millions of pounds from public funds devoted to it, there has been insufficient convergence of medical practice as most audit loops have not been closed. Various reasons have been given by doctors to explain or excuse their failure to adopt uniform practices. These include lack of time, the claim that medicine is an art and the assertion that standards infringe clinical freedom.

One of the hallmarks of geriatric medicine is the emphasis placed on how diseases affect function. The geriatrician routinely considers the extent to which pathology is compromising the patient's ability to live independently by assessing mental and physical function and social circumstances. Standardized assessment scales for the elderly are available and have been recommended jointly by the British Geriatrics Society and the Royal College of Physicians but not one of them is used routinely in all departments of geriatric medicine.

Guidelines of good practice from which standards can be set are being produced by many professional bodies. The protection offered by national guideljnes is that the only difference between providets will be cost. Contracting choices should then be made on the basis of efficiency, as quality will be uniform.

Medical culture is being forced to change by the contracting process. Doctors might be reluctant to follow clinical guidelines but the National Health Service Management Executive is expecting them to be used in the annual contracting round and has initially selected seven, which purchasers are recommended to use as a basis for negotiating contracts in 1994/5. As more guidelines are produced they too will have to be incorporated into contracts. Providers will have to demonstrate that patients are being managed according to the agreed guidelines. Care which routinely exceeds that laid down by the guidelines will be more expensive and render that practitioner uneconomic. If care fails to achieve the standard, purchasers are likely to consider placing contracts elsewhere. If contracts are not renewed, income will drop and jobs will go. Thus, in order to survive Trusts will have to insist that the doctors they employ standardize their work. Consultants who do not care for patients to the agreed standards will be invited to practice their version of 'clinical freedom' elsewhere. The consultant who not only practices according to accepted guidelines but also attracts extra business may qualify for a performance related bonus.

The routine use of guidelines will require changes in what and how medical students and doctors in training are taught. Teaching should be 


\section{Editorial}

based on nationally accepted clinical guidelines. Examinations should test the candidates' ability to apply the guidelines appropriately. Registration with the General Medical Council should include the requirement to practice in accordance with guidelines.
Those who believe that quality is optional are likely to find that the condition is terminal.

Rebecca B Dunn, Consultant Geriatrician, St Martin's Hospital, Midford Road, Bath BA2 5RP, $U K$. 